\title{
Récits sur l'origine des langues : enromancier Babel
}

\author{
Marie-Pascale Halary*
}

\section{Narrativas sobre a origem das línguas: romanceando Babel}

\section{Resumo}

O estudo trata da narrativa da Torre de Babel e se interessa pelas primeiras traduções do texto (séculos XII e XIII). Depois de destacar as principais características da exegese desta passagem, o estudo se concentra em uma tradução original, a saber, a Gênesis de Evrat (final do século XII). Pergunta-se, portanto, como esse texto constrói o valor do francês, enquanto a interpretação comum da narrativa babeliana contribui para o estabelecimento de uma hierarquia linguística?

Palavras-chave: Babel, traduções, língua francesa.

\section{Narratives about the origin of languages: romanticizing Babel}

\section{Abstract}

This study deals with the story of the Tower of Babel and aims at look the early translations (twelfth and thirteenth centuries) of this text. After outlining the main characteristics of its exegesis, it examines an original translation of the story: the Genèse by Evrat (end of the twelfth century). How does this text value the French language whereas the common interpretation of the Babel story plays a part in linguistic hierarchy?

Keywords: Babel, translations, French language.

* Docteure de l'Université Paris IV-Sorbonne. Maîtresse de conférences en langue et littérature médiévales à l'Université Lumière-Lyon 2.

Email: marie-pascale.halary@univ-lyon2.fr 


\section{Récits sur l'origine des langues : enromancier Babel}

\section{Résumé}

Cette étude, qui porte sur le récit de la Tour de Babel, s'intéresse aux premières traductions de ce texte (douzième et treizième siècles). Après avoir mis en évidence les principales caractéristiques de l'exégèse de ce passage, elle se concentre sur une traduction originale : la Genèse d'Evrat (fin du XII ${ }^{e}$ siècle). Comment ce texte construit-il la valeur du français alors que l'interprétation commune du récit babélien participe de la mise en place d'une hiérarchie linguistique?

Mots-clés : Babel, traductions, langue française.

Si elle fut moins tardive que ce qu'on lit parfois ${ }^{1}$, la rencontre entre la Bible et la langue romane n'avait rien d'une évidence à une époque où la traduction vernaculaire du texte sacré faisait peser une menace sur le monopole linguistique (et herméneutique) exercé par les clercs. Et, de fait, bien que les histoires de la Bible française reviennent en général sur les expériences de translations faites ou commandées à date ancienne par des figures comme Lambert le Bègue ou Pierre Valdès (que l'historiographie associe à des mouvements qui s'établissent aux marges de l'institution), elles ne manquent pas, non plus, de rappeler différentes interdictions ou condamnations portant sur la mise en roman du texte sacré. Au XII siècle, selon un exemple bien connu, il ne faut pas moins que l'apparition de la Vierge thaumaturge pour autoriser, pour ainsi dire, la traduction-adaptation des Écritures par Hermann de Valenciennes : en ce Moyen Âge central, la rencontre de la langue vernaculaire et de la Bible reste de l'ordre du «miracle $»^{3}$. Dès lors, avant même de s'interroger sur les modalités selon lesquelles la « littérature » dite profane se nourrit des échos du texte sacré, il convient peut-être de revenir sur le geste symbolique que représente le transfert de la Vulgate latine dans cet idiome que les textes cléricaux désignent comme la lingua vulgaris ${ }^{4}$.

1 «Une opinion assez répandue veut que les premières traductions bibliques françaises remontent à Lefèvre d'Etaples (1525). C'est à peu près exact si l'on entend traduction au sens où l'entendent les philologues depuis la Renaissance ; c'est profondément inexact si, respectant les catégories médiévales, on consent à ne pas séparer anachroniquement les deux démarches complémentaires de la traduction et du commentaire » (Hasenohr, 1990, p. 323).

2 Sur la question des traductions médiévales de la Bible, voir surtout Bogaert (1964) et Nobel (2011).

3 Herman de Valenciennes, Li Romanz de Dieu et de sa mère, 1. 60-68, p. 175-177.

4 Pour un développement, selon une perspective très différente de celle qui sera développée ici, de certains problèmes liés à la traduction du texte sacré, voir Frye (1984, p. 41 sq.). 
Or, « adapter et traduire suppose une réflexion sur les langues, dont les traces se révèlent en particulier dans la Genèse où la dénomination est importante à chaque étape de la création $\|^{5}$ : si l'on en croit l'exégèse médiévale, cette «question linguistique » est particulièrement thématisée dans l'épisode de la tour de Babel (Gen 11, 1-9). Selon l'interprétation commune, le récit de la construction de l'édifice puis de la punition divine établit en quelque sorte, à l'intérieur même du texte biblique, les conditions d'impossibilité de sa propre vernacularisation ${ }^{6}$. En raison de cette dimension réflexive, c'est donc le dossier des réécritures et des interprétations de Babel que je voudrais rouvrir ici.

Si le corpus latin des reprises de Babel et de ses commentaires a été bien étudié ${ }^{7}$, tel n'est pas le cas du corpus constitué par les mises en roman de l'épisode : il s'agira d'examiner l'effet produit par la rencontre entre la langue vernaculaire et le récit babélien, lieu habituel d'une légitimation de la supériorité du latin. Le passage se prête-t-il autant que d'autres extraits génésiaques à une translation romane ? Les versions françaises reprennentelles les principales lignes interprétatives de la tradition exégétique latine - au risque d'accueillir en leur sein le principe même de leur dévalorisation - ou introduisent-elles dans le champ des discours sur les langues quelques éléments inédits ? S'il est toujours délicat de repérer l'irruption de la nouveauté, je voudrais montrer qu'il est un texte français, la mise en roman d'Evrat (fin XII ${ }^{\mathrm{e}}$ siècle), qui, dans le contexte discursif des $\mathrm{XII}^{\mathrm{e}}$ et $\mathrm{XIII}^{\mathrm{e}}$ siècles, se distingue par des caractéristiques étonnantes.

\section{Approche sérielle}

Afin de percevoir la singularité de cette réécriture d'Evrat et d'en mesurer le degré d'inventivité, il convient de commencer par revenir sur la tradition interprétative autour de Babel, à partir de la construction d'une série : l'analyse, selon la proposition de Jérôme Baschet, «vise[ra] à intégrer dans la régularité d'une série, la singularité de chaque œuvre - qui combine des réponses particulières à un ensemble d'options possibles, et qui noue un réseau propre de tensions et de contradictions $»^{8}$.

\footnotetext{
5 Ducos (2012, p. 50).

${ }^{6} \quad$ Voir d'abord et avant tout Lusignan (1986). Pour une approche plus générale, qui ne se limite pas au Moyen Âge, voir également Céard (1980) et Dauphiné (1996).

Outre Lusignan (1986), voir surtout Borst (1957-1963) et Grondeux (2005b, p. 49-51).

8 Baschet (1996, p. 116).
} 
Comme l'ont montré les études portant sur l'exégèse de Babel' ${ }^{9}$, les commentaires du passage s'inscrivent dans la continuité de ceux d'Augustin ${ }^{10}$ et s'organisent autour de plusieurs constantes. Ils rappellent notamment que la perte de l'unité linguistique originelle est le châtiment divin consécutif à une faute (l'orgueil) ; cette confusion voulue par Dieu est traditionnellement mise en relation avec le nom de Babylone. De cette punition, « avènement des langues dans l'histoire ${ }^{11}$, naissent 72 langues et nations tandis que la " langue commune primitive $»^{12}$, l’hébreu, est conservée grâce à Héber et sa descendance. Ainsi, dans la Cité de Dieu :

quand un orgueil impie valut aux nations d'être punies et divisées par la diversité des langues, et à la cité des impies de recevoir le nom de « confusion », c'està-dire de Babylone, il y eut une famille, celle d'Héber, destinée à conserver la langue que tous parlaient auparavant. [...] Et comme sa famille, lors de la division des nations en langues différentes, conserva la sienne - laquelle était d'abord commune à tout le genre humain, comme on le croit non sans raison - cette langue fut depuis appelée « hébraïque ». Car il fallut la distinguer des autres par un nom propre, comme les autres aussi d'ailleurs. Quand elle était seule, elle n'avait pas de nom particulier : c'était la «langue humaine » ou « langage humain », que parlait seul tout le genre humain ${ }^{13}$.

9 Voici le texte de la Vulgate : Erat autem terra labii unius, et sermonum eorumdem. Cumque proficiscerentur de oriente, invenerunt campum in terra Sennaar, et habitaverunt in eo. Dixitque alter ad proximum suum: Venite, faciamus lateres, et coquamus eos igni. Habueruntque lateres pro saxis, et bitumen pro camento : et dixerunt: Venite, faciamus nobis civitatem et turrim, cujus culmen pertingat ad calum : et celebremus nomen nostrum antequam dividamur in universas terras. Descendit autem Dominus ut videret civitatem et turrim, quam adificabant filii Adam, et dixit : Ecce, unus est populus, et unum labium omnibus : caperuntque hoc facere, nec desistent a cogitationibus suis, donec eas opere compleant. Venite igitur, descendamus, et confundamus ibi linguam eorum, ut non audiat unusquisque vocem proximi sui. Atque ita divisit eos Dominus ex illo loco in universas terras, et cessaverunt adificare civitatem. Et idcirco vocatum est nomen ejus Babel, quia ibi confusum est labium universa terra : et inde dispersit eos Dominus super faciem cunctarum regionum (Gen 11, 1-9).

10 Grondeux (2005a).

11 Dragonetti (2006, p. 266).

12 Prima illa communis (Augustin, La Cité de Dieu, trad. G. Combès, XVI, XI, 2, p. 225). Sur la conception de l'hébreu comme langue première, voir en particulier Dahan (1995).

13 Augustin, La Cité de Dieu, trad. G. Combès, XVI, XI, 1, p. 223 : quando merito elatioris impietatis gentes linguarum diversitate punitae atque divisae sunt et civitas impiorum confusionis nomen accepit, hoc est, appellata est Babylon, non defuit domus Heber, ubi ea quae antea fuit omnium lingua remaneret. [...] Quia ergo in eius familia remansit haec lingua, divisis per alias linguas ceteris gentibus, quae lingua prius bumano generi non inmerito creditur fuisse communis, ideo deinceps Hebraea est nuncupata. Tunc enim opus erat eam distingui ab aliis linguis nomine proprio, sicut aliae quoque vocatae sunt nominibus propriis. Quando autem erat una, nibil aliud quam bumana lingua vel bumana locutio vocabatur, qua sola universum genus humanum loquebatur (p. 222). 
Pour Augustin comme pour une grande partie des commentateurs médiévaux, l'opposition entre deux paradigmes (unité linguistique / intercompréhension / état antérieur au péché versus multiplicité linguistique / confusion / conséquence du péché) sert la construction d'une véritable hiérarchie linguistique dans laquelle l'hébreu, langue adamique originelle, est la langue la plus éminente. Un autre passage scripturaire, la mention des trois langues sur la croix (Jean 19, 20), consacre la dignité de deux autres idiomes, le grec et le latin ${ }^{14}$. Ce sont donc des épisodes bibliques ${ }^{15}$ et avant tout celui de Babel qui fondent la représentation médiévale des langues.

Mais, comme l'a montré Anne Grondeux, le récit babélien n’est pas seulement celui qui assigne à la différenciation linguistique une origine brutale et divine ; contrairement à ce que laisse parfois penser l'historiographie moderne qui construit à son tour un «mythe du mythe de Babel $»^{16}$, ce récit est aussi un obstacle à toute réflexion sur l'évolution et la variation des langues - le continum historique entre le moment du châtiment et les langues médiévales n'étant jamais envisagé :

Aucun exégète ne déclare par exemple formellement que le français, l'allemand, l'anglais sont issus de Babel, de même qu'aucun ne cherche à répertorier les 72 langues parmi celles de son temps. Il n'y a donc pas de connexion pratique entre ce que l'on infère de Babel et la situation contemporaine des commentateurs ${ }^{17}$.

Autrement dit, si l'épisode génésiaque fait de la diversité linguistique les conséquences d'une punition, il va jusqu'à empêcher toute conception diachronique des langues.

Dans un tel contexte, le geste qui consiste à traduire le récit latin de la Vulgate en langue dite vulgaire n'est pas anodin et il n'est sans doute pas très surprenant que la fiction romane des $\mathrm{XII}^{\mathrm{e}}$ et $\mathrm{XIII}^{\mathrm{e}}$ siècles accueille peu de réécritures babéliennes ${ }^{18}$ (moins, par exemple, que les reprises vernaculaires du

14 Lusignan (1986, p. 59-61), Grondeux (2005b, p. 51-53). Sur ces trois langues sacrées, voir aussi Bourgain (2005, p. 28-31).

15 Pour les épisodes importants sur cette question linguistique, voir aussi la nomination des animaux par Adam, le reniement de Pierre et le récit de la Pentecôte.

16 Grondeux (2005a, p. 667).

17 Ibid., p. 669.

18 Ainsi, si le Roman d'Alexandre ou le roman de toute chevalerie de Thomas de Kent évoque, à la faveur du déplacement d'Alexandre depuis l'Inde jusqu'à la Perse, la ville de Babylone, la tour de Babel et le felon Nemrod, rien n'est dit concernant les langues. On apprend seulement qu'à cause de la folie du roi, son lignage est « devis par les terres » (v. 7849). 
Cantique des cantiques qui, selon la lecture médiévale, est pourtant destiné à un public monastique ${ }^{19}$ ) ; de même, il n'est peut-être pas étonnant que certaines versions françaises de la Bible puissent passer l'épisode sous silence ${ }^{20}$. C'est que l'épisode babélien, éventuellement associé à l'interprétation d'autres passages scripturaires, contribue à la valorisation des trois langues sacrées :

Et a la verité dire, devant ce que la tour Babel fust faite, tout home avoient une meisme parleure naturelement, c'est ebreu; mais puis que la diversités des langues vint entre les homes, sor les autres en furent .iii. sacreees, ebrieu, grieu, latin. (Brunet Latin, Li Livres dou Tresor, III, 1, 3, p. 317)

Tout se passe comme si la rupture linguistique introduite par la punition divine permettait non seulement de valoriser l'hébreu, langue paradisiaque et native, mais aussi, pour la période placée sous le signe de la pluralité des langues, de distinguer un groupe de trois langues, supérieures aux autres ${ }^{21}$. Dans ce système de représentations, on ne s'étonnera donc pas de trouver relativement peu de reprises de Babel dans la langue dite vulgaire.

Dans la plupart des cas, au demeurant, le choix linguistique ne modifie pas profondément l'interprétation de l'épisode. Comme dans les commentaires latins, l'édification de la tour provoque une rupture : les hommes passent de l'unité à la diversité linguistique et l'analyse ne se prolonge pas au-delà de cette modification radicale.

Devant n'avoit ou monde que.i. langaige, / Setante et.ij. en fut par cel outraige ; / Par lour forfait qu'il orent commancié, / Furent les laingues et parolles changiés. (Bible anonyme du Ms. Paris, BNF fr. 763, v. 1198-1201)

[...] [Diex] mua lor lengage, / Qui n'estoit que .i. seux jadis, / Et diex en fist .lx. et .x.. (Macé de la Charité, Bible, I, Genèse, Exode, v. 1190-1192)

De même, à la manière des textes exégétiques, le geste des hommes est attribué à leur orgueil et/ou, depuis Flavius Josèphe, à celui de Nemrod, " qui tant d'orguio en son cour hot, / De nonsen et de felonie » (Macé de la Charité, Genèse, v. 1204-1205). La différenciation des langues, comme dans le texte de la Vulgate, introduit de la confusion et empêche toute

\footnotetext{
19 Sur les échos de ce texte dans la littérature médiévale, je me permets de renvoyer à mon étude : Halary (2016).

20 Voir par exemple Herman de Valenciennes, Li Romanz de Dieu et de sa mère : l'œuvre compile des extraits bibliques et para-bibliques.

21 Là encore, je renvoie aux analyses de Lusignan (1986).
} 
intercompréhension : Venite igitur, descendamus, et confundamus ibi linguam eorum, ut non audiat unusquisque vocem proximi sui. Grâce à l'emprunt confusion, les textes français vont même jusqu'à reproduire le jeu verbal de la Vulgate qui associe le nom de Babel au participe confusum (Et idcirco vocatum est nomen ejus Babel, quia ibi confusum est labium universa terra ${ }^{22}$. Ainsi de Macé de la Charité qui reprend l'Aurora de Pierre Riga :

Babiloyne est 'confusion' / Par droite interpretacion, / Et la tor qui est li aus leux / Nous note les cours orgoilleux. (Macé de la Charité, Genèse, v. 1215-1218)

La Bible d'Acre qui, pourtant, développe moins l'épisode que les Bibles françaises en vers, reprend, quant à elle, le rapprochement étymologique de la Vulgate dans deux gloses marginales ${ }^{23}$, respectivement appelées par les termes Babiloine et Babel:

Babiloine est confusion por ce que les lengues y furent confuzes et departies [Babel] Qui est orres apelee Babiloine por le departement des gens qui est toz jorz car ne poent estre en acort ${ }^{24}$

Si ces explications ne font pas sens en français (pas plus qu'en latin), leur reprise témoigne de la fidélité des réécritures vernaculaires vis-à-vis de la tradition babélienne. Je n'ai trouvé qu'un seul texte qui s'efforce d'expliquer le jeu étymologique autour de Babel/Babylone et de l'adapter à la langue latine :

Aucuns l'apelent le tour Babel de «balbucio » en latin, qui vaut a dire baboillier, les autres le tour de Baldac; en moult de diverses mennieres le nommerent diverses gens. De celle muanche des langages fu le terre et le pays vironnés apelés et est encore Babilon, de ce mot «babilon », qui vaut a dire confusion de langue, dont je vous ay parlé. (Placides et Timéo on Le secrés as philosophes, \411)

Dans les versions françaises, les variations les plus notables concernent les petites saynètes qui apportent des détails sur la construction de

\footnotetext{
22 Comme le rappelle Benoît Grévin, « Le latin de la Vulgate ne rend pas le vieux calembour qui transformait la pieuse étymologie suméro-babylonienne Ka-dingir-ra/Bâb-ilân̂̂ ("Porte des Dieux") en sa parodie hébraïque Babel (“confusion”)» (2012, p. 123).

23 Sur ces gloses marginales, voir Nobel (2006, p. XXXVII-XXXIX).

24 Ibid., p. 13-14.
} 
l'édifice ${ }^{25}$ ou qui, précisément, donnent à voir la confusion introduite par la diversification linguistique :

Li uns parloit d'unne menniere et l'autre d'autre, et ne s'entendoient point, car il furent soudainement de .LXIX. langues et, quant li uns demandoit yaue, li autres li aportoit pierre et li autres mortier, et li autres entendoit qu'i li deist folie ; si commencha entre euls contens merveilleux et grant descort et grant merlee. (Placides et Timéo ou Le secrés as philosophes, \ 410)

Dans tous ces épisodes, on le constate, l’interprétation générale du récit génésiaque reste la même. Même si c'est à partir de l'exégèse de Babel que se construit en partie la hiérarchie linguistique médiévale et que, de ce fait, l'épisode scripturaire peut sembler se prêter moins que d'autres à une mise en roman, la version vernaculaire de Babel ne diffère guère de la tradition latine (dont, au demeurant, elle s'inspire largement). Autrement dit, quand le récit rencontre la langue vernaculaire, contrairement à ce que pourrait laisser penser le «mythe du mythe de Babel », la différence linguistique ne semble pas être une variable hautement significative : c'est une même série que composent les reprises latines et romanes.

\section{Un « vulgaire illustre » français?}

Cette conjoncture discursive, marquée par une forte régularité, est à même de faire ressortir l'« inventivité » de la version proposée par Evrat. Le début du passage s'inscrit pourtant dans le prolongement des autres réécritures babéliennes, comme l'Historia scolastica dont l'auteur s'inspire très largement. S'agissant de la représentation des langues, on note tout d'abord la valorisation habituelle de l'hébreu en tant que langue native :

Cist languages estoit hebreuz / Puis cele hore que Damerdeuz / Ot fait Adam a sa faiture ; / Sachiés que nule creature / N'avoit parlé se l'ebreu non : / Hebreus est de mult grant renon! (Evrat, Genèse, v. 2708-2713)

De même, cette version met en scène l'incompréhension entre les hommes ${ }^{26}$ après que Dieu a procédé à la différenciation des langues :

\footnotetext{
25 «Ajostent pieres por faire fondement / Quatre vins toises de lé, et de lonc .c., / Et la maisiere nonante et .ij. de lé, / Itant ou plus en i out compassé. / De sablon font et de chauz le mortier / De plusors terres i viennent li ovrier» (Bible anonyme du Ms. Paris, BNF fr. 763 , v. 1163-1168).

26 Sur ce point, voir aussi la reprise de l'association entre Babel et confusion, inspirée ici par Pierre le Mangeur : Evrat, Genèse, v. 2891-2896.
} 
Damerdeus mult tost s'en venja / Mais altrement nes laidenja / Ke li uns l'altre n'entendoit. / Et cant cist morter demandoit / Se li aparelhoit cil tiele ; / Cist redescendoit par eschiele / Cant il devoit en halt monter / Ne cil ne pooit reconter / A cestui rien qu'il puisse entendre, / Ki l'en deüst ardoir u pendre ; / Bien huchoient a halte vois, / Mais tot ce ne valoit dous nois, / Cant les langues erent diverses / Des vilz gens males et disperses / Ki contre Deu welent aler. (Evrat, Genèse, v. 2832-2846)

Cette diversité linguistique, au demeurant, s'oppose à l'unité et l'immutabilité du latin :

La tor que cil quiderent faire / Est a sainte Eglise contraire, / C'ausi fu ele a Damerdeu. / Sainte Eglise muet de tel leu / Ke toz jors valut et valdra, / Ja ses languages ne faldra / N'iert ja ne changiéz ne muéz. (Evrat, Genèse, v. 2880-2886)

Concernant la conduite du récit et les grandes lignes interprétatives de l'épisode, la version d'Evrat ne se distingue donc pas vraiment de la série des reprises babéliennes. Elle témoigne même peut-être de la connaissance de certains problèmes canoniques concernant l'exégèse du passage. Les commentateurs s'interrogent en effet sur le pluriel de la Vulgate : descendamus, et confundamus ibi linguam eorum. Parmi les lectures possibles, certains suggèrent de voir dans ce propos une adresse de Dieu aux anges ${ }^{27}$. Tel est précisément le choix du texte d'Evrat qui ajoute cette précision et lève l'ambiguïté :

Mult s'en corocha nostre sire, / A poi que tos ne les confont ; / Mult li poise de ce qu'il font. / As angles dist : "Confundons les / Toz, si cum il sunt, demanés. » (Evrat, Genèse, v. 2827-2831)

On ne peut pas exclure non plus que l'insistance du texte sur le mouvement de chute ${ }^{28}$ soit destinée à construire un diptyque entre le récit de la Chute et celui de Babel : c'est là une association fréquente de l'exégèse médiévale ${ }^{29}$.

\footnotetext{
Voir par exemple la Glose ordinaire : Biblia cum glossa ordinaria (1481).
}

28 Par exemple : «Mult les fist de halt avaler : / Ki trop halt monte, trop halt chiét » (Evrat, Genèse, v. 2847-2848).

29 «Le péché de Babel est pour l'histoire linguistique ce qu'est le péché originel pour l'histoire civile et politique : ce qui a éloigné l'homme de l'Un et l'a fait entrer dans la région de la “dissemblance” »(Rosier-Catach, 2011, p. 47). 
Dans le cadre de cette réécriture globalement topique de l'épisode babélien, la version d'Evrat inclut toutefois un développement beaucoup moins habituel ${ }^{30}$.

[...] Issi cum vos savéz, / Ki maint language oï avéz, / Chascune terre, vilz et chiere, / A son language, a sa maniere. / Les cruelz terres les ont durs / Et fors et griés et trop obscurs, / Téz que nus n’i ose habiter / Por vendre ne por achater. / De quelque part que l'en les prange / Tuit sunt et divers et estrange / Fors que li languages franchois : / C'est cil que Deus entent anchois, / K'il lo fist et bel et legier / Sel puet l'en croistre et abregier / Mielz que toz les altres languages, / Ce dist li cortois et li sages. (Evrat, Genèse, v. 2856-2871)

Ces vers se distinguent avant tout parce qu'ils établissent explicitement une continuité entre Babel, épisode fondateur du plurilinguisme selon le système de représentations médiéval, et le moment de l'énonciation. Le narrateur s'adresse directement aux lecteurs-auditeurs et il fait appel à leur expérience linguistique : «vos [....] / Ki maint language oï avéz». Le point commun entre l'épisode de l'Ancien Testament et cette échappée vers le présent de la réception est la diversité linguistique. Les vers qui précèdent ce petit développement reviennent en effet sur la juste punition divine que constitue la différenciation des langues :

Bien furent tuit li mal vengié / Cant li language sunt cangié. / Piece a que l'en dist et retrait / K'en ne puet joïr de sorfait. / De cestui s'est Deus corociéz / Ses a toz des langues bleciéz. / Bleciés ? (Evrat, Genèse, v. 2850-2856)

Une situation similaire caractérise donc les deux moments, celui de l'histoire vétéro-testamentaire et celui de l'énonciation : c'est la multiplicité des langues. «a la tor furent devisé / Trestuit li language qui sunt / Et qui cuerent par tot li mont!» (Evrat, Genèse, v. 2735-2737), dit ailleurs Evrat. En d'autres termes, si on s'appuie sur les analyses d'Anne Grondeux déjà évoquées, cette traduction-adaptation de la Genèse est relativement exceptionnelle car elle fait de Babel la cause et l'explication du pluralisme linguistique observable au moment de la composition - et ce texte est d'autant plus exceptionnel que c'est en français qu'il établit cette continuité historique. Ce dernier point n'est peut-être pas sans rapport avec le deuxième fait notable

30 Dans l'étude qu'il joint à son édition, Wil Boers déclare ne pas connaître de sources pour un tel passage (Boers, 2002, livre II, t. 3, p. 167-168). 
de ce développement : alors que, conformément à l'exégèse, Evrat définit la pluralité linguistique comme la conséquence de la faute des hommes, châtiés par Dieu, il propose un étonnant éloge du français. Les qualités du language franchois sont d'ordre esthétique (il est « et bel et legier») et le lexique rhétorique ("sel puet l'en croistre et abregier») montre la dignité de cette langue littéraire, à même de se prêter à l'amplificatio et l'abbreviatio. Plus encore, dans ce contexte babélien, la prééminence de cette langue vis-à-vis de Dieu est propre à construire son extraordinaire valeur.

[Le français] bénéficie surtout d'un privilège théologique : le français est la langue entendue de Dieu. Curieux coup de force qui profite de Babel pour élever une langue vernaculaire au rang de langue élue, de langue du Père ${ }^{31}$.

On le voit, les procédés de valorisation du français (beauté d'une langue qui est, pour ainsi dire, d'origine divine) ne sont pas sans rappeler les modalités par lesquelles les médiévaux construisent l'excellence de l'hébreu ou celle du latin. Enfin, le dernier élément d'importance, qui sous-tend l'ensemble de ce petit développement, est la substitution d'une pensée de la diversité ou de la variété (sinon de la variatio) à une pensée de la confusio: «De quelque part que l'en les prange / Tuit sunt et divers et estrange ». Le texte note une forme de correspondance entre les différences géographiques et climatiques et les différences socio-économiques et linguistiques ${ }^{32}$ : à chaque lieu la langue qui lui est propre. Dans ces vers qui prolongent le récit génésiaque, ce n’est plus tout à fait la confusion post-babélique ; c'est presque déjà la conception, visible chez Dante, d'« une variabilité accidentelle, imputable à la variété des usages selon les lieux différents où [la langue] est parlée $»^{33}$.

Ce qui est manifeste, c'est donc l'inventivité ou l'originalité de cette réécriture de Babel au sein de la tradition latine et française. Non seulement le texte d'Evrat proclame la beauté du français - et c'est sans doute une des premières attestations de la construction de sa qualité esthétique ${ }^{34}-$ mais, en outre, il proclame cette beauté dans le cadre d'une reprise de Babel, récit dans lequel les médiévaux lisent précisément la diversité linguistique comme le résultat d'un châtiment divin.

\footnotetext{
$31 \quad$ Fritz (2016, p. 200).

32 Irène Rosier-Catach (Rosier-Catach, 2011, p. 117) note qu'on trouve déjà des manifestations de ce topos chez Virgile et Augustin.

33 Imbach et Rosier-Catach (2005, p. 525). Sur cette question, voir l'ensemble de cet article.

34 Je me permets de renvoyer à Halary (2013).
} 
Ce renversement interprétatif par rapport à la signification commune de Babel s'adosse, dans le prologue de la Genèse d'Evrat, à la valorisation du geste du traducteur qui met en roman la Vulgate latine. Selon un procédé qu'on retrouve ailleurs, Evrat se pose comme le continuateur d'un autre traducteur, Jérôme, qui

De l'ebreu en latin la mist, / Com cil ki bien s'en entremist. / S'Evraz a ce se puet prometre / K'il la repusse en romans metre / Si ke l'entendent cler et lai, / N'a pas mis sa paine en delai. / Lui semble que c'est biens a faire / Qu'en i mete le miliaire / Del tens qu'ele est encommencie / Et del latin enromancie. (Evrat, Genèse, v. 103-112)

La figure prestigieuse de saint Jérôme, qui est régulièrement sollicitée, contribue donc à légitimer l'opération de traduction et de mise en roman. Mais il semble que la promotion du français, dans le passage consacré à Babel, aille beaucoup plus loin. Le choix de la langue romane ne permet pas seulement de prolonger le travail de Jérôme et de rendre le texte sacré accessible aux clercs et aux laïcs ; le français est valorisé pour lui-même et il est placé au sommet d'une hiérarchie qui englobe d'autres langues (les langues estranges c'est-à-dire les autres vernaculaires ?) : «Sel puet l'en croistre et abregier / Mielz que toz les altres languages, / Ce dist li cortois et li sages ». Il est peut-être permis de s'interroger sur la mention finale du courtois et du sage. Même si le substantif courtois, associé à sage, a évidemment un sens éthique, son origine montre que cette voix est socialement située. C'est une voix à la fois sage et courtoise qui décrète la valeur esthétique du français. Et, de fait, en ce Moyen Âge central, la promotion de la langue dite vulgaire, par sa mise en écrit notamment, est bien ce qui sert le système de valeurs de l'aristocratie : si le latin est la langue des clercs, la scripturalité romane est en passe de devenir la marque distinctive des grands laïcs.

Bien que cette promotion du français soit seulement esquissée dans la Genèse d'Evrat, ces éléments ne sont pas sans faire écho au texte que Dante rédige en latin en 1304-1305 pour " défendre et illustrer » la langue vulgaire. De la même façon, en effet, le De vulgari eloquentia s'appuie sur une reprise originale du récit babélien où la variation linguistique n'est pas le châtiment divin punissant l'orgueil des hommes ${ }^{35}$, où le latin de vient pas de l'hébreu ${ }^{36}$ et où la quête du «vulgaire illustre » permet d'entendre cet éloge du français :

\footnotetext{
35 Voir surtout Rosier-Catach (2011) et Imbach et Rosier-Catach (2005).

36 Voir surtout Lusignan (1986, p. 45).
} 
La langue d'oïl allègue ainsi en sa faveur que, grâce à la nature plus facile et plus agréable de son vulgaire, tout ce qui est rédigé ou inventé en prose vulgaire lui appartient en propre, à savoir la Bible compilée avec les faits des Romains et des Troyens, les très belles aventures du roi Arthur, et bien d'autres ouvrages d'histoire et de science ${ }^{37}$.

Un peu plus d'un siècle après Evrat, le poète florentin reconnaît à la fois la valeur intrinsèque du language franchois et celle de sa "littérature », c'est-à-dire des productions écrites composées dans cette langue. Pour Dante, toutefois, le « vulgaire illustre » ne peut s'identifier avec la langue d'oïl et c'est, logiquement, l’italien qui est donné comme la plus excellente des langues :

Nous appelons illustre, cardinal, palatin et curial ce vulgaire d'Italie qui appartient à toute cité italienne et ne semble être d'aucune ${ }^{38}$.

Et Dante de préciser dans un développement qui peut rappeler la mention du « courtois » chez Evrat :

À bon droit il doit être appelé « curial », parce que la curialité n'est rien d'autre qu'une règle bien pondérée de ce qui doit être fait. Et comme la balance d'une telle pesée ne se trouve habituellement que dans les plus excellentes des cours, il s'ensuit que tout ce qui, dans nos actions, est bien pondéré est appelé «curial». Et par conséquent, puisque ce vulgaire a été pesé dans la plus excellente des cours des Italiens, il mérite d'être appelé « curial » ${ }^{39}$.

En français comme en latin, pour la langue d'oïl comme pour l'italien, il semble bien que "l'émancipation littéraire des vulgaires ${ }^{40}$ se conquiert aussi grâce à une "promotion sociolinguistique et idéologique » ${ }^{41}$ : malgré Babel, la belle langue est peut-être d'abord celle qu'on parle à la cour.

\footnotetext{
37 Dante, De l'éloquence en vulgaire, I, X, p. 121 : Allegat ergo pro se lingua oïl, quod propter sui faciliorem ac delectabiliorem vulgaritatem quicquid redactum sive inventum est ad vulgare prosaycum, suum est : videlicet Biblia cum Troianorum Romanorumque gestibus compilata et Arturi regis ambages pulcerrime et quamplures alie ysortie ac doctrine (p. 120).

38 Ibid., p. 159 : dicimus illustre, cardinale, aulicum et curiale vulgare in Latio quod omnis latie civitatis est et nullius esse videtur (p. 158).

39 Ibid., p. 165 : Est etiam merito curiale dicendum, quia curialitas nil aliud est quam librata regula eorum que peragenda sunt : et quia statera buiusmodi librationis tantum in excellentissimis curiis esse solet, binc est quod quicquid in actibus nostris bene libratum est, curiale dicatur. Unde, cum istud in excellentissima Ytalorum curia sit libratum, dici curiale meretur (p. 164).

40 Bourgain $(2005$, p. 32).

41 Grévin (2012, p. 394).
} 
Dans le système de représentations médiéval où l'épisode de Babel contribue à construire la supériorité de l'hébreu et du latin, enromancier Babel peut apparaître comme une singulière gageure puisqu'il s'agit de translater dans l'idiome vernaculaire le passage même qui proclame l'excellence des trois langues sacrées. Il semble bien que cette tension aboutisse à un évitement relatif : les mises en roman de Babel ne sont pas très nombreuses aux XII ${ }^{\mathrm{e}}$ et XIII ${ }^{\mathrm{e}}$ siècles, en particulier dans la littérature de fiction qui, à l'instar du Roman de Renart, ne répugne pourtant pas à accueillir des mises en roman du texte sacré et, en particulier, du texte génésiaque. Quant aux versions françaises de la Bible qui réécrivent l'épisode de la Tour, elles s'inscrivent assez facilement dans la tradition exégétique latine et cléricale, dont elles découlent : le choix du français ne modifie pas l'interprétation commune et le changement linguistique ne semble pas être problématique. De fait, la reprise scripturaire se place dans une chaîne de réécritures et de commentaires, qu'il convient de répéter. Comme le note Anne Grondeux à partir d'un corpus latin, le mythe de Babel est loin de susciter une comparaison avec la situation linguistique médiévale.

Il est toutefois un texte, la Genèse d'Evrat, qui se distingue doublement. D'une part, à l'échelle de la série des reprises de l'épisode biblique (dont le clerc champenois retient pourtant les grandes lignes interprétatives), il propose une valorisation du language franchois, assez inédite pour une période si ancienne et tout à fait surprenante dans ce contexte babélien. Ce texte est par ailleurs étonnant si on considère le diptyque bien connu entre l'épisode de Babel et celui de la Pentecôte. Curieusement, c'est ici le récit de la confusion des langues qui permet l'éloge d'une langue dite vulgaire.

Peut-être est-ce parce que, plus que l'intelligibilité du vernaculaire et plus que sa capacité à diffuser le message chrétien, ce que la Genèse d'Evrat met en valeur, c'est la qualité esthétique (voire sociale) du français, langue quasi divine. Il se peut que ce soit là la première «institution du français $»^{42}$ comme la «plus belle des langues».

42 Je reprends la formule de Renée Balibar (1985). 


\section{Bibliographie}

\section{Bibliographie primaire}

Augustin, La Cité de Dieu, trad. G. Combès, Paris, Institut d’Études augustiniennes, 1996.

Bible d'Acre (La) : Genèse et Exode, éd. P. Nobel, Besançon, Presses universitaires de Franche-Comté, 2006.

Bible anonyme du Ms. Paris, BNF fr. 763, éd. J. C. Szirmai, Amsterdam, Rodopi, 1985.

Biblia cum glossa ordinaria Walafridi Strabonis aliorumque et interlineari Anselmi Laudunensis, Adolf Rusch pro Antonio Koberger [Strasbourg 23.IX.1481] : en ligne : https://archive. thulb.uni-jena.de/ufb/rsc/viewer/ufb derivate 00000064/Inc 831 00047.tif (consulté le 30 septembre 2019).

Brunet Latin, Li Livres dou Tresor, éd. F. J. Carmody, Genève, Slatkine Reprints, 1998 [1948].

Dante, De l'éloquence en vulgaire, introduction et appareil critique par I. Rosier-Catach, trad. A. Grondeux, R. Imbach et I. Rosier-Catach, Paris, Fayard, 2011.

Evrat, Genèse, éd. W. Boers, Leiden, Université de Leiden, 2002.

Herman de Valenciennes, Li Romanz de Dieu et de sa mère, éd. I. Spiele, Leyde, Presse universitaire de Leyde, 1975.

Macé de la Charité, Bible, I, Genèse, Exode, éd. J. R. Smeets, Leiden, Universitaire Pers Leiden, 1967.

Placides et Timéo ou Le secrés as philosophes, éd. C. Thomasset, Genève, Droz, 1980.

Thomas de Kent, Le Roman d'Alexandre ou le roman de toute chevalerie, éd. B. Foster et I. Short, trad. C. Gaullier-Bougassas et L. Harf-Lancner, Paris, Champion, 2003.

\section{Bibliographie secondaire}

BALIBAR R., L'Institution du français. Essai sur le colinguisme des Carolingiens à la République, Paris, Presses universitaires de France, 1985.

BASCHET J., "Inventivité et sérialité des images médiévales. Pour une approche iconographique élargie », Annales. Histoire, Sciences Sociales, 51, 1, p. 93-133, 1996.

BOGAERT P.-M., "Bible française », dans Dictionnaire des lettres françaises, Le Moyen Âge, édition revue et mise à jour par G. Hasenhor et M. Zink, Paris, Fayard, 1964, p. 179-197.

BORST A., Der Turmbau von Babel. Geschichte der Meinungen über Ursprung und Vielfalt der Sprachen und Völker, Stuttgart, A. Hiersemann, 1957-1963, 6 vol.

CÉARD J., « De Babel à la Pentecôte : la transformation du mythe de la confusion des langues au XVI ${ }^{\mathrm{e}}$ siècle ", Bibliothèque d'humanisme et Renaissance, 42, 3, p. 577-594, 1980. 
DAHAN G., " Nommer les êtres : exégèses et théories du langage dans les commentaires médiévaux de Genèse 2, 19-20 », dans S. Ebbesen, Sprachtheorien in Spätantike und Mittelalter, Tübingen, G. Narr, 1995, p. 55-74.

DAUPHINÉ J., « Le mythe de Babel », Babel, 1, p. 163-173, 1996.

DRAGONETTI R., "Dante face à Nemrod : Babel mémoire et miroir de l’Eden ? ", dans Ch. Lucken, Dante, la langue et le poète. Recueil d'études, Genève, Belin, 2006, p. 257-275.

DUCOS J., «La langue d'Adam », dans A. P. Bagliani, Adam le premier homme, Florence, Sismel, 2012, p. 49-68.

FRITZ J.-M., « Conjointures troyennes. Pierre le Mangeur, Chrétien de Troyes et Evrat, auteur du poème de la Genèse », dans V. Fasseur et J.-R. Valette, Les Écoles de pensée du XII ${ }^{\mathrm{e}}$ siècle et la littérature romane (oc et oïl), Turnhout, Brepols, 2016, p. 195-209.

FRYE N., Le Grand Code. La Bible et la littérature, préface de T. Todorov, trad. C. Malamoud, Paris, Seuil, 1984 [1981].

GRÉVIN B., Le Parchemin des cieux. Essai sur le Moyen Âge du langage, Paris, Seuil, 2012. GRONDEUX A., « La question du langage avant 1200 », Mélanges de l'École Française de Rome, Moyen Âge, 117, 2, p. 665-695, 2005.

GRONDEUX A., «Le latin et les autres langues au Moyen Âge : contacts avec des locuteurs étrangers, bilinguisme, interprétation et traduction (800-1200)», dans E. Bury, Tous vos gens à latin. Le latin, langue savante, langue mondaine ( $\mathrm{XIV}^{\mathrm{e}}-\mathrm{XVII}^{\mathrm{e}}$ siècles), Genève, Droz, 2005, p. 47-67.

HALARY M.-P., "Revendiquer la "beauté littéraire" dans les romans du XII siècle », publié le 16 mars 2013 dans le cadre du projet dirigé par Hélène Merlin, «La beauté », sur le site Transitions : www.mouvement-transitions.fr/intensites/la-beaute.

HALARY M.-P., " "Ge sui noire, mais ge sui bele” : en français dans le texte », dans M. Barsi et A. Preda, Le Cantique des cantiques dans les lettres françaises, Milan, Edizioni Universitarie di Lettere Economia Diritto, 2016, p. 71-89.

HASENOHR G., «Traductions et littérature en langue vulgaire » dans H.-J. Martin et J. Vezin, Mise en page et mise en texte du livre manuscrit, Paris, Cerf, Édition du Cercle de la librairie-Promodis, p. 229-352, 1990.

IMBACH R. et ROSIER-CATACH I., « De l'un au multiple, du multiple à l'un : une clé d'interprétation pour le De vulgari eloquentia », Mélanges de l'École Française de Rome, Moyen Âge, 117, 2, p. 509-529, 2005.

LUSIGNAN S., Parler vulgairement. Les intellectuels et la langue française aux XIII ${ }^{\mathrm{e}}$ et XIV $^{\mathrm{e}}$ siècles, Paris/Montréal, Vrin/Presses de l'université de Montréal, 1986.

NOBEL P., «La traduction biblique », dans C. Galderisi, Translations médiévales. Cinq siècles de traductions en français au Moyen Âge ( $\mathrm{xI}^{\mathrm{e}}-\mathrm{xv}^{\mathrm{e}}$ siècles). Étude et répertoire, Turnhout, Brepols, 2011, vol. 1, p. 207-223.

Submetido em: 24-10-2019

Aceito em: 21-11-2019 